\title{
Advection-diffusion-mortality solutions for investigating pelagic larval dispersal
}

\author{
A. E. Hill \\ School of Ocean Sciences, University of Wales, Bangor, Marine Science Laboratories, Menai Bridge, Gwynedd LL59 5EY, \\ United Kingdom
}

\begin{abstract}
Many marine organisms release into the ocean planktonic larvae which must reach specific sites in order to be recruited to the next stage of their life-cycle. Solutions of the 2-dimensional advection-diffusion-mortality equation are presented which enable larval losses between release and recruitment sites to be quantified. The model assumes constant mean advection, eddy diffusivity and larval mortality. Solutions confirm that dispersal can make a significant contribution to pelagic losses and that survival is highly sensitive to the level of mean advection. The solutions have been used to examine the minimum fecundity required to overcome pelagic larval losses from a population.
\end{abstract}

\section{INTRODUCTION}

Many marine organisms, including fish, crustaceans and molluscs, have complex life-cycles in which the early stages take the form of planktonic larvae. During this planktonic phase, larvae are dispersed from their release site by a combination of currents (horizontal advection) and random eddy-like motions at a variety of space and time scales (turbulent diffusion).

Although the distribution of organisms is controlled by pelagic processes during dispersal, the point at which larvae are released (the spawning sites) is frequently a fairly well-defined site to which adults return year after year. Not only are larvae released from known regions but there is also commonly an imperative that they reach a second spatially well-defined site (a nursery or recruitment area) at the end of the larval phase in order to proceed to the next stage of the lifecycle. Between release and nursery sites, however, planktonic larvae may be lost from the system because of mortality and dispersal processes.

There are many examples of the requirements of marine organisms for specific sites at the end of a planktonic phase. North Sea plaice Pleuronectes platessa, for example, are spawned over wide areas of the English Channel and the Southern and German Bights of the North Sea. At the end of their larval stage, however, they settle specifically into shallow, sandy areas, most notably the Wadden Sea (van der Veer et al. 1990). Similarly Bay of Biscay sole Solea solea are spawned in off-shore continental shelf waters with muddy or sandy substrate. Larvae are dispersed inshore and require an estuarine environment at the end of the larval period (Koutsikopoulos et al. 1989). Numerous other examples are to be found in the volume edited by Blaxter et al. (1989) which concerns the early life history of fish.

For other species the locations of spawning and recruitment sites are sometimes even more clearly defined. Some species, particularly benthic organisms, are substrate-specific. Nephrops norvegicus, for example, is a benthic decapod which burrows in a substrate of fine mud. Larvae that are released from mud areas must ultimately settle back onto mud to continue with the juvenile part of the life-cycle (Hill 1990). Many substrate-specific shore species, such as barnacles, release larvae into the pelagic environment. At the end of their larval phase, barnacles must be located in the vicinity of a suitably hard or rocky surface upon which to settle and continue their life-cycle. Common also are estuarine species which release larvae into estuaries and which are subsequently flushed onto the open continental shelf. Recruitment to the juvenile phase, however, requires that later larval stages return to the vicinity of an estuary. Blue crab Callinectes sapidus, discussed by Epifanio et al. (1989) and Goodrich et al. (1989), is a good example of such an organism.

The purpose of this paper is to present some simple solutions of the 2-dimensional advection-diffusionmortality equation in order to quantify losses of larvae 
between well-defined release and recruitment sites. The solutions are based on idealised configurations of release and recruitment sites and the model is deficient in a number of important respects. Nevertheless the solutions have 3 possible uses. Firstly they can be used to obtain first order answers to real problems when it is not practicable to use more sophisticated numerical models. Secondly the solutions can be used as a simple, but physically grounded, parameterization of dispersal processes in more complex models which include the pelagic phase as a component part. Finally the (exact) analytical solutions presented here may be used as test solutions for numerical models of advection-diffusion processes. The results presented here are a generalisation of solutions discussed by Hill (1990) which were developed for the particular case of Nephrops norvegicus. These general results also include, as a special case, diffusion away from a coastline discussed by Beverton \& Holt (1957).

The advection-diffusion-mortality equation and some of its simple solutions are first discussed. These solutions are then incorporated into a simple closed life-cycle model to illustrate the relationship between larval dispersal and fecundity.

\section{TWO-DIMENSIONAL ADVECTION-DIFFUSION- MORTALITY MODEL}

It should be noted that the term larvae will be used a little loosely in the following sections. This is because there is a range of points in the very early parts of the life-cycle when pelagic dispersal commences. Sometimes dispersal starts at the egg stage, but for other organisms fertilized eggs are retained by the female and only released at a later stage of larval development. The term larvae will henceforth be used to describe all planktonic early life-stages released into the water column.

In order to describe the spreading of larvae from a release site in a way which is readily amenable to solution, attention is restricted at the outset to the case of 2-dimensional dispersal. No attempt is made, therefore to describe vertical variations in any quantities whether they be velocity or turbulence fields or the vertical distribution of larvae. Neglect of vertical processes is potentially a serious limitation of the model. In particular it does not permit the interaction between vertical larval migration and vertical current structure to be directly included. There is, however, evidence from a number of species that systematic use of vertical current shear is used to exert some control upon horizontal movement. With this limitation in mind, the model should either be applied to organisms which do not migrate vertically or to early larval stages where vertical migration is not well developed. Alternatively the larval concentrations given by the model can be regarded as depth-averaged values resulting from depth-averaged advection, diffusion and vertical-migration processes. In this way the net effect of interaction between vertical migration and current shear is a net displacement velocity which can be considered as subsumed into the values given to the current velocity. Adopting the depth-averaged approach is far from satisfactory, however, and no substitute for ultimately using a fully 3-dimensional model. For the present, however, in the interests of obtaining tractable solutions, a 2-dimensional approach is retained.

The depth-integrated concentration, $C$, of larvae at any point depends only upon its horizontal space coordinate $(x, y)$ and time $t$, thus

$$
C=C(x, y, t)
$$

and is in units of numbers of larvae per unit area. The concentration of larvae at any point in space at any time, $t$, after release is governed by the advectiondiffusion-mortality equation

$$
\frac{\partial C}{\partial t}+u \frac{\partial C}{\partial x}+v \frac{\partial C}{\partial y}=K_{x} \frac{\partial^{2} C}{\partial x^{2}}+K_{y} \frac{\partial^{2} C}{\partial y^{2}}-m C
$$

which is a slightly more general form of the equation discussed by Hill (1990). Here $u$ and $v$ are the $x$ and $y$ components of depth-mean current velocity and are assumed to be constant over the entire region of interest. $K_{x}$ and $K_{y}$ are the turbulent diffusion coefficients in the $x$ and $y$ directions and are also taken to be constant. Diffusion for which $K_{x}$ and $K_{y}$ are constant is known as Fickian diffusion (Fischer et al. 1979). Physically it represents the state in which the size of a diffusing patch of material is greater than the scale of the turbulent eddy motions responsible for the diffusion. Fickian diffusion is appropriate for the description of larvae spread over large scales. The quantity $m$ in Eq. (1) is the instantaneous larval mortality rate and represents the continual loss of larvae as they die within the water column due to predation and starvation.

Provided that $u, v, K_{x}, K_{y}$ and $m$ are constant, analytical solutions for $C$ and Eq. (1) may be obtained subject to a variety of initial and boundary conditions. The quantity that is of particular interest in the present study is not $C$ but the total number of larvae that are found in a given region of space after they have been in the water column, for a time $\tau$. The region over which larvae are released (at $t=0$ ) into the water column will be referred to as the larval release site (site LR) (Fig. 1). The region over which larvae will be counted at time $\tau$ will be referred to as the recruitment site (site R). Here the term recruitment refers to transition from larval to juvenile phases. The number of larvae present at the recruitment site at $t=\tau$ is denoted by $N_{\tau}$. The recruit- 
Fig. 1. Definition figure for the model. Larvae are released from site LR. The site at which larvae must be located at the end of the planktonic period is termed the recruitment site (site $\mathrm{R})$ and is centred at the origin of coordinates. Site LR is positioned arbitrarily with respect to site $\mathrm{R}$
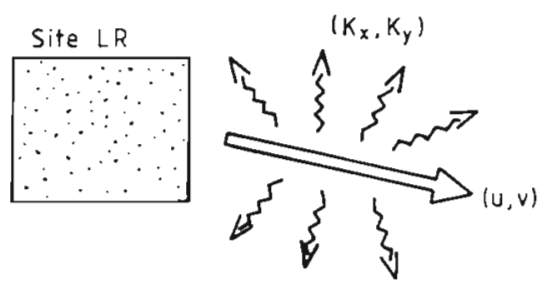

y

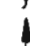
v)$$
\text { (n) }
$$

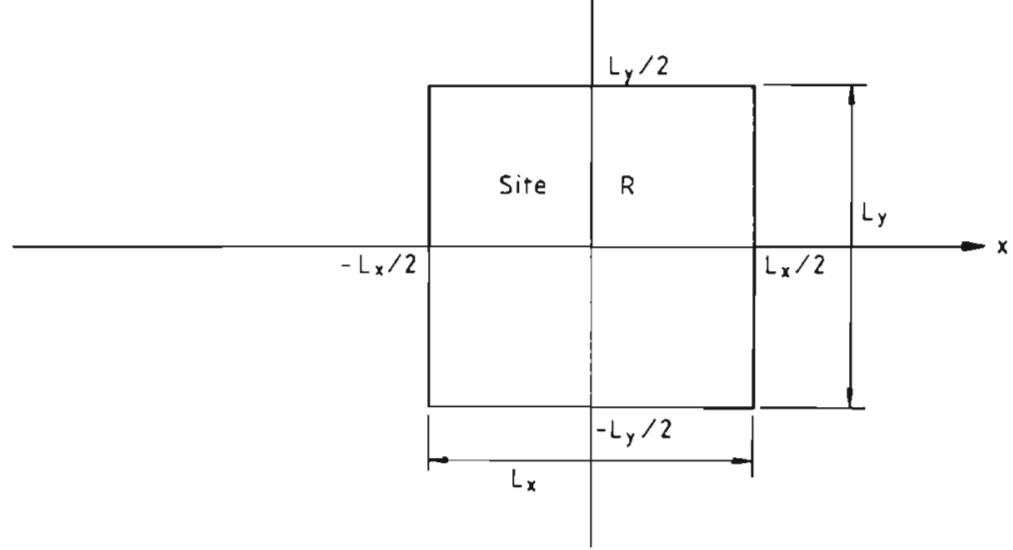

$$
C(x, y, 0)=N_{0} \delta\left(x-x_{\mathrm{p}}\right) \delta\left(y-y_{\mathrm{p}}\right)
$$

coordinates $(x=0, y=0)$ and is taken as a rectangular region defined by

$$
\begin{aligned}
& -L_{x} / 2 \leq x \leq L_{x} / 2 \\
& -L_{y} / 2 \leq y \leq L_{y} / 2
\end{aligned}
$$

and has dimension $L_{x}$ in the $x$ direction and $L_{y}$ in the $y$ direction.

The number of larvae present within this region at time $\tau$ is given by

$$
N_{\tau}=\int_{-L_{Y} / 2}^{L_{Y} / 2} \int_{-L_{X} / 2}^{L_{X} / 2} C(x, y, \tau) \mathrm{d} x \mathrm{~d} y
$$

Three sets of solutions for $C(x, y, \tau)$ and $N_{\tau}$ are given below.

\section{Spike release}

First consider the case when $N_{0}$ larvae are released at $t=0$ as a 'spike' of infinite concentration at a point $\left(x_{\mathrm{p}}\right.$ $\left.y_{p}\right)$ as depicted in Fig 2 . Note that the release point $\left(x_{p}\right.$. $y_{p}$ ) can be located anywhere with respect to the recruitment site. Suppose that the region into which larvae disperse is unbounded so that material can spread to infinity in all directions given enough time. A solution of Eq. (1) under such circumstances will be applicable to the release of larvae at a point in the open sea. The solution has been discussed by Hill (1990) and is reproduced here as a special case of a more general solution to follow. At time $t=0$ the initial concentration is given by where $\delta$ is the Dirac delta function that represents a spike. The concentration of larvae at any arbitrary point $(x, y)$ is given at a later time $\tau$ by

$$
\begin{aligned}
C(x, y, \tau)= & \frac{N_{0}}{4 \pi \sqrt{K_{x} K_{y}} \tau} e^{-m \tau} \exp \left[-\frac{\left(x-x_{\mathrm{p}}-u \tau\right)^{2}}{4 K_{x} \tau}\right] \times \\
& \exp \left[-\frac{\left(y-y_{\mathrm{p}}-v \tau\right)^{2}}{4 K_{y} \tau}\right]
\end{aligned}
$$

and is a well-known result from diffusion theory. The value of $N_{\tau}$ as obtained by substituting Eq. (4) into Eq. (2) is less well known and is derived by Hill (1990) and repeated here in a slightly more general form

$N_{\tau}=\frac{N_{0} e^{-m \tau}}{4}\left[\operatorname{erf}\left(\alpha_{1}\right)+\operatorname{erf}\left(\alpha_{2}\right)\right]\left[\operatorname{erf}\left(\beta_{1}\right)+\operatorname{erf}\left(\beta_{2}\right)\right]$

where

$$
\begin{array}{ll}
\alpha_{1}=\frac{L_{y}-2 y_{\mathrm{p}}-2 v \tau}{4 \sqrt{K_{y} \tau}} & \alpha_{2}=\frac{L_{y}+2 y_{\mathrm{p}}+2 v \tau}{4 \sqrt{K_{y} \tau}} \\
\beta_{1}=\frac{L_{x}-2 x_{\mathrm{p}}-2 u \tau}{4 \sqrt{K_{x} \tau}} & \beta_{2}=\frac{L_{x}+2 x_{\mathrm{p}}+2 u \tau}{4 \sqrt{K_{\mathrm{x}} \tau}}
\end{array}
$$

and $\operatorname{erf}(x)$ is the error function defined by

$$
\operatorname{erf}(x)=\frac{2}{\sqrt{\pi}} \int_{0}^{x} \exp \left(-\varepsilon^{2}\right) d \varepsilon
$$

for any arbitrary variable $\varepsilon$. The error function arises quite naturally in problems involving diffusion and recurs in the more general solutions that follow. 


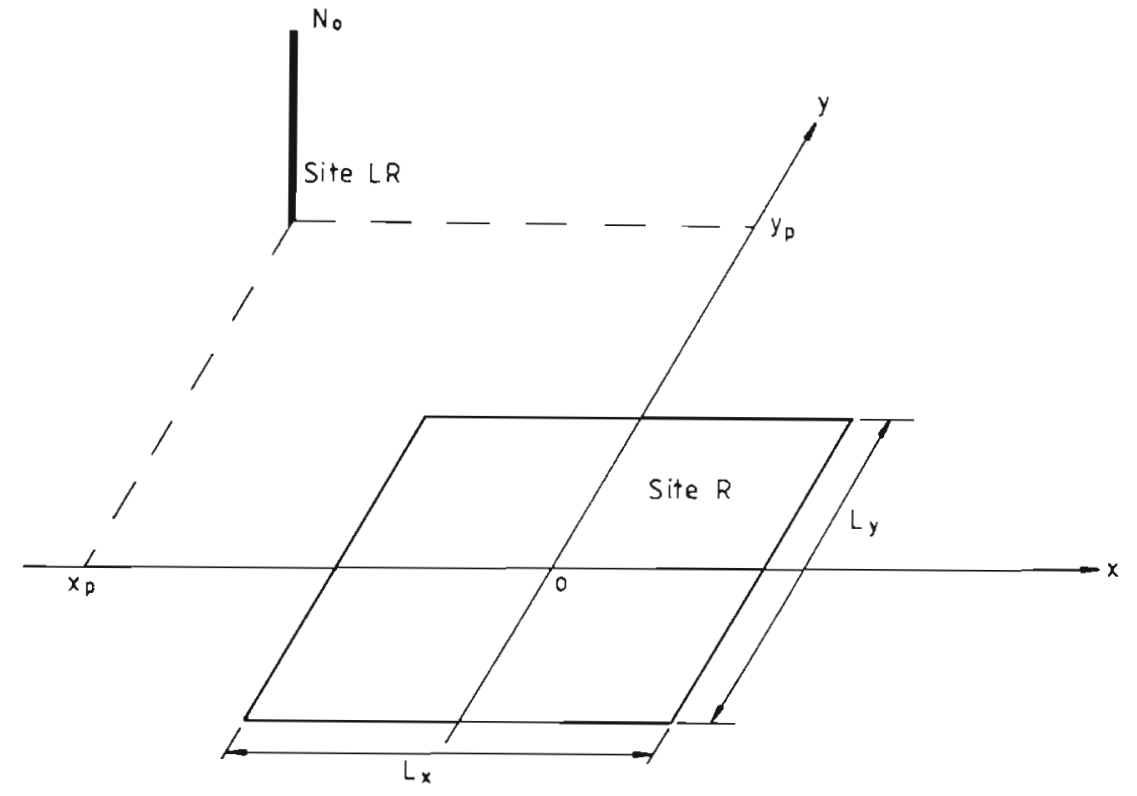

Fig. 2. The case of larval release in a spike of infinite concentration at an arbitrary point $\left(x_{\mathrm{p}}, y_{\mathrm{p}}\right)$. The release point may be located within site $\mathrm{R}$ or outside it
Eq. (5) can be rewritten as

$$
N_{r}=\alpha e^{-m \tau} N_{0}
$$

where $\alpha$ represents the fraction of larvae that reach the recruitment site when losses are due to dispersal processes alone $(m=0)$. Consequently the fraction of larvae that are lost to the recruitment site because of dispersal is

$$
V_{\mathrm{L}}=1-\alpha
$$

and is termed the larval vagrancy following Sinclair (1988). From Eq. (5) it follows that

$$
V_{\mathrm{L}}=1-\frac{1}{4}\left[\operatorname{erf}\left(\alpha_{1}\right)+\operatorname{erf}\left(\alpha_{2}\right)\right]\left[\operatorname{erf}\left(\beta_{1}\right)+\operatorname{erf}\left(\beta_{2}\right)\right]
$$

\section{Release from a rectangular site}

A spike function may be used to represent a highly localized release of larvae. More commonly, however, larvae will be released over a wider area. Hill (1990) described how release of larvae over a broad region could be built up as the sum of many spike releases over the region. A general solution of Eq. (2) for release over a rectangular region can, however, be found as follows.

Consider the rectangular region shown in Fig. 3 . It is defined by coordinates $X_{1}, X_{2}$ in the $x$ direction and $Y_{1}$. $Y_{2}$ in the $y$ direction. The dimensions of the rectangular region are $D_{x}=X_{2}-X_{1}$ and $D_{y}=Y_{2}-Y_{1}$ in the $X$ and $y$ directions respectively. This rectangular release site is arbitrarily located with respect to the recruitment site and may either be within the recruitment region or be separated from it depending upon the values assigned to $X_{1}, X_{2}, Y_{1}, Y_{2}$. It is assumed that $N_{0}$ larvae are released uniformly from this site at $t=0$. The initial concentration of larvae is thus

$$
C(x, y, 0)=C_{0}=\frac{N_{0}}{D_{x} D_{y}}=\text { constant }
$$

The region into which larvae disperse is assumed to be unbounded and thus represents an open sea release. It can be shown that the concentration of larvae at any point $(x, y)$ at subsequent time $\tau$ is given by

$$
\begin{aligned}
C(x, y, \tau)= & \frac{C_{0}}{4} \mathrm{e}^{-m \tau}\left[\operatorname{erf}\left(\varepsilon_{2}\right)-\operatorname{erf}\left(\varepsilon_{1}\right)\right] \times \\
& {\left[\operatorname{erf}\left(\delta_{2}\right)-\operatorname{erf}\left(\delta_{1}\right)\right] }
\end{aligned}
$$

where

$$
\begin{array}{ll}
\varepsilon_{1}=\frac{x-u \tau-X_{1}}{2 \sqrt{K_{x} \tau}} & \varepsilon_{2}=\frac{x-u \tau-X_{2}}{2 \sqrt{K_{x} \tau}} \\
\delta_{1}=\frac{y-v \tau-Y_{1}}{2 \sqrt{K_{y} \tau}} & \delta_{2}=\frac{y-v \tau-Y_{2}}{2 \sqrt{K_{Y} \tau}}
\end{array}
$$

Eq. (12) can be integrated using Eq. (2) to obtain the total number of larvae, $N \tau$, present at the recruitment site at time $\tau$. This calculation involves integrating the error function which can be done using the result

$$
\int \operatorname{erf}(x) \mathrm{d} x=x \operatorname{erf}(x) \frac{1}{, \pi} \exp \left(-x^{2}\right)
$$

The result of the integration is

$$
N_{r}=\frac{N_{0}}{D_{x} D_{y}} \sqrt{K_{x} K_{y}} \tau \mathrm{e}^{-m r} F(\tau) G(\tau)
$$


Fig. 3. The case of larval release which is distributed uniformly over a rectangular site with dimensions $D_{x}, D_{y}$. The release site may be within or outside the recruitment site

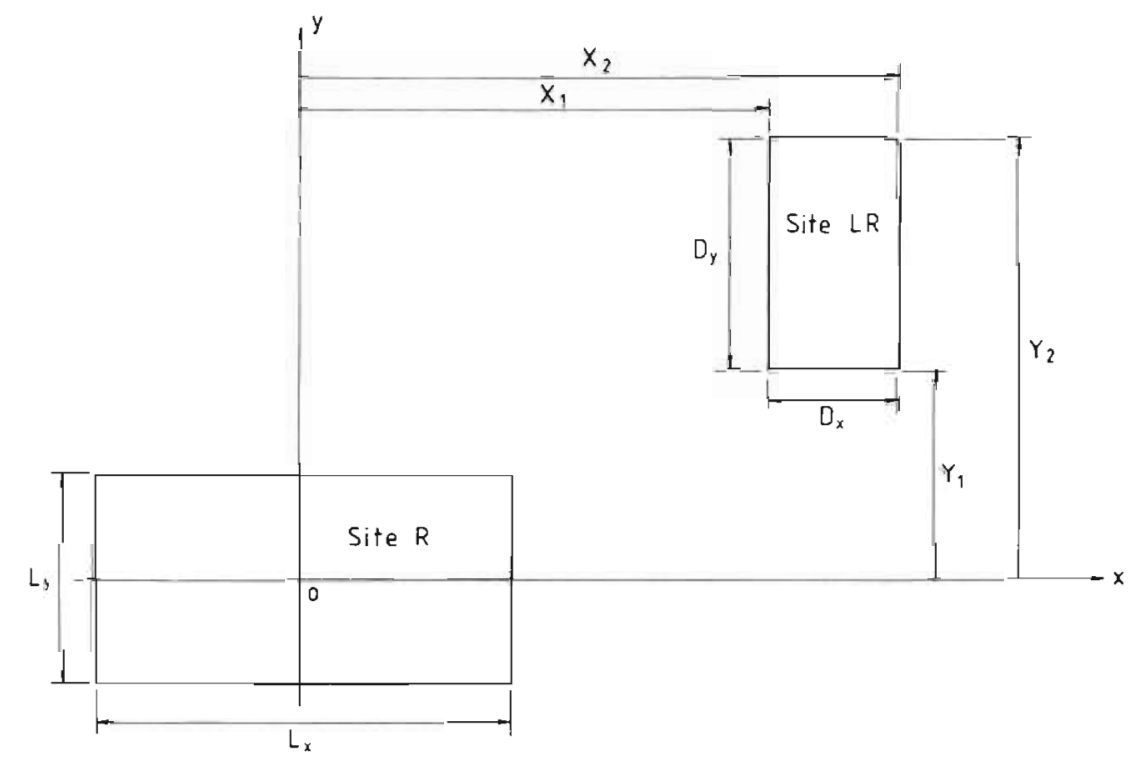

where the functions $F$ and $G$ are given by

$$
F(\tau)=\int_{a_{1}}^{a_{2}} \operatorname{erf}(\varepsilon) \mathrm{d} \varepsilon-\int_{b_{1}}^{b_{2}} \operatorname{erf}(\varepsilon) \mathrm{d} \varepsilon
$$

and

$$
G(\tau)=\int_{c_{1}}^{c_{2}} \operatorname{erf}(\varepsilon) \mathrm{d} \varepsilon-\int_{d_{1}}^{d_{2}} \operatorname{erf}(\varepsilon) \mathrm{d} \varepsilon
$$

with

$$
\begin{array}{ll}
a_{1}=-\frac{\left(L_{x}+2 u \tau+2 X_{1}\right)}{4 \sqrt{K_{x} \tau}} & a_{2}=\frac{\left(L_{x}-2 u \tau-2 X_{1}\right)}{4 \sqrt{K_{x} \tau}} \\
b_{1}=-\frac{\left(L_{x}+2 u \tau+2 X_{2}\right)}{4 \sqrt{K_{x} \tau}} & b_{2}=\frac{\left(L_{x}-2 u \tau-2 X_{2}\right)}{4 \sqrt{K_{x} \tau}} \\
c_{1}=-\frac{\left(L_{y}+2 v \tau+2 Y_{1}\right)}{4 \sqrt{K_{y} \tau}} & c_{2}=\frac{\left(L_{y}-2 v \tau-2 Y_{1}\right)}{4 \sqrt{K_{y} \tau}} \\
d_{1}=-\frac{\left(L_{y}+2 v \tau+2 Y_{2}\right)}{4 \sqrt{K_{y} \tau}} & d_{2}=\frac{\left(L_{y}-2 v \tau-2 Y_{2}\right)}{4 \sqrt{K_{y} \tau}}
\end{array}
$$

Although the above set of results (Eqs. 15 to 18) appear rather formidable at first sight, the expression for $N_{\tau}$ in Eq. (15) is readily evaluated by a computer program in only a few lines of code. The expressions in Eq. (16) and Eq. (17) are evaluated using Eq. (14). A standard computer library subroutine can be used to evaluate erf $(x)$.

It can be shown that the result Eq. (15) has the sensible property that

$$
N_{\tau} \rightarrow N_{0} \text { as } \tau \rightarrow 0
$$

Furthermore, as the rectangular release site is shrunk $\left(D_{x}, D_{y} \rightarrow 0\right)$ the solution given by Eq. (15) approaches the special case solution of a spike release given by Eq. (5).
The larval vagrancy is

$$
V_{\mathrm{L}}=1-\alpha=1-\frac{\sqrt{K_{x} K_{y}}}{D_{x} D_{y}} \tau F(\tau) G(\tau)
$$

\section{Release from a site in the vicinity of a coastline}

The solutions for spike release and release from a rectangular site have both assumed that larvae may disperse into an unbounded region of open sea. If a coastal boundary is present, however, dispersal will be inhibited because clearly there can be no flux of larvae through the coast. Under suitable conditions, nonetheless, the solution in the presence of a coastline can be obtained by a simple manipulation of the solution for the unbounded case.

Consider a long, straight coastline parallel to the $x$ axis and situated at $y=-y_{c}$ as shown in Fig 4. Larvae are released from the rectangular site (LR) in Fig. 4. The presence of the coastline imposes 2 boundary conditions. The first is that there can be no flow of water perpendicular to the coast at $y=-y_{\mathrm{c}}$. Because $u$ and $v$ are assumed to be constant everywhere over the region this means that $v=0$ everywhere. Thus only flow parallel to the coastline (with speed $u$ ) is permitted. The second condition imposed by the coast is that there can be no diffusive flux of larvae in the $y$ direction at $y=-y_{\mathrm{c}}$. The latter condition requires that

$$
\frac{\partial C}{\partial y}=0 \quad \text { at } y=-y_{c}
$$

A solution which satisfies the advection-diffusionmortality equation (Eq. 1) and the boundary condition Eq. (20) can be found using a technique known as the 


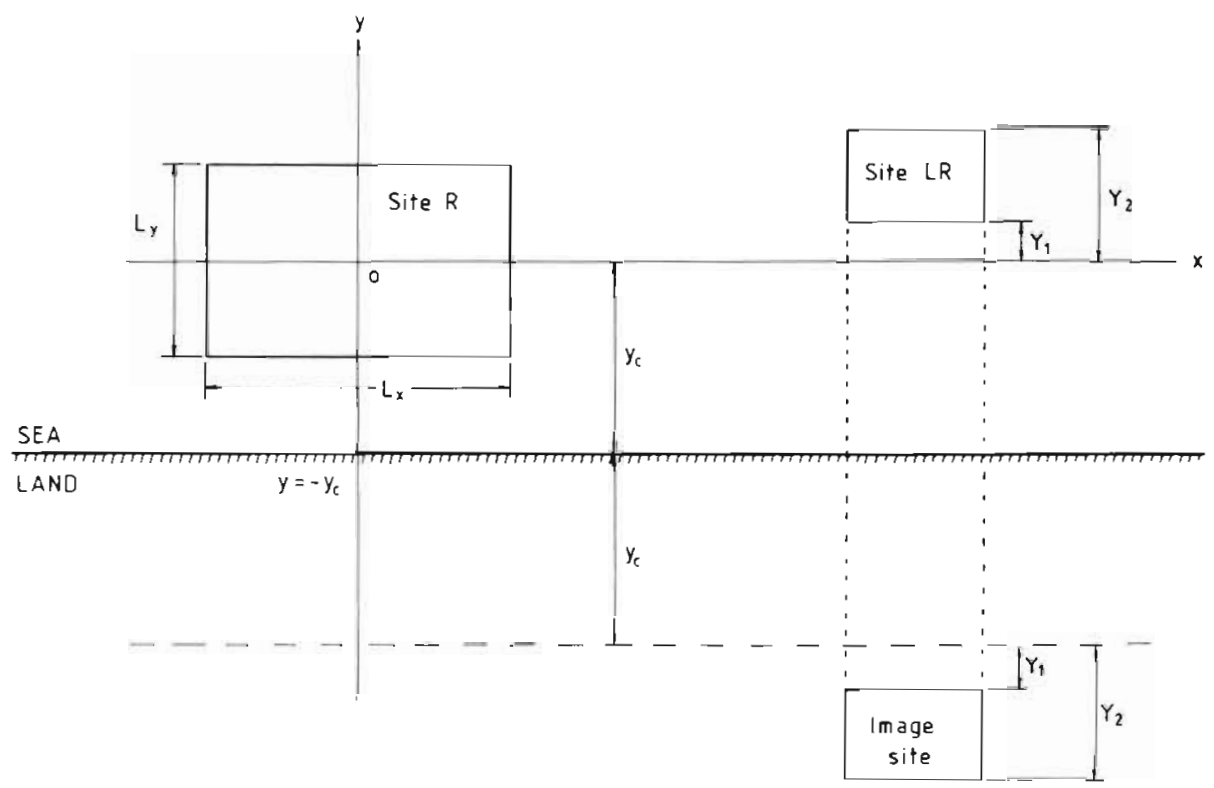

Fig. 4. The case of larval release in the vicinity of a long, straight coastline. The release site is again a rectangular region with dimensions $D_{x}, D_{y}$ and larvae are released uniformly over the site

method of images. The method involves finding a solution for a release of larvae at a site which is in the mirror image position when the site LR is reflected in the coastline (note that in reality such a site would be inland and thus has no physical or biological meaning in its own right!). If. however, the mirror image solution is added to the solution given by Eq. (15) for an unbounded region the resulting solution satisfies both the advection-diffusion-mortality equation and the boundary condition Eq. (20) and is therefore the required solution. The image solution is itself easily obtained by replacing $Y_{1}$ in Eq. (12) by $-\left(2 y_{c}+Y_{1}\right)$ and replacing $Y_{2}$ by $-\left(2 y_{c}+Y_{2}\right)$. The solution for dispersal in the vicinity of the coastline is therefore

$$
N_{\tau}=\frac{N_{0} \sqrt{K_{x} K_{Y}}}{D_{X} D_{Y}} \tau \mathrm{e}^{-m \tau} F(\tau)\left[G(\tau)+G_{\lambda}(\tau)\right]
$$

where $F$ and $G$ are as defined previously and

$$
G_{i}(\tau)=\int_{c_{11}}^{c_{12}} \operatorname{erf}(\varepsilon) \mathrm{d} \varepsilon-\int_{d_{11}}^{d_{12}} \operatorname{erf}(\varepsilon) \mathrm{d} \varepsilon
$$

and

$$
\begin{aligned}
& c_{i 1}=-\frac{\left[L_{y}+2 v \tau-2\left(2 y_{c}+Y_{1}\right)\right]}{4 \sqrt{K_{y} \tau}} \\
& c_{i 2}=\frac{\left[L_{y}-2 v \tau+2\left(2 y_{c}+Y_{1}\right)\right]}{4 \sqrt{K_{y} \tau}} \\
& d_{i 1}=-\frac{\left[L_{y}+2 v \tau-2\left(2 y_{c}+Y_{2}\right)\right]}{4 \sqrt{K_{y} \tau}} \\
& d_{i 2}=\frac{\left[L_{y}-2 v \tau+2\left(2 y_{c}+Y_{2}\right)\right]}{4 \sqrt{K_{y} \tau}}
\end{aligned}
$$

Here the subscript $i$ refers to the image site. In this case the larval vagrancy, $V_{\mathrm{L}}$, is

$$
V_{\mathrm{L}}=1-\frac{\sqrt{K_{\mathrm{x}} K_{y}}}{D_{x} D_{y}} \tau F(\tau)\left[G(\tau)+G_{i}(\tau)\right]
$$

\section{APPLICATIONS AND LIMITATIONS OF THE SOLUTIONS}

The advection-diffusion-mortality solutions for $N_{\tau}$, Eq. (5), Eq. (15) and Eq. (21) provide a basis for quantitative estimates of larval losses due to vagrancy and mortality between release and recruitment sites. Furthermore these solutions may be used to parameterize these processes in more general models which include the pelagic phase as a component part. Fig. 5 shows how some common configurations of release and recruitment sites can be idealized in order to apply the solutions derived above.

Fig. $5 \mathrm{a}$ shows the case when the larval release site (LR) and the recruitment site (R) coincide. This configuration was applied by Hill (1990) to the case of Norway lobster Nephrops norvegicus, a benthic organism which lives on patches of fine, cohesive mud. $N$. norvegicus releases larvae into the water column which, after a pelagic larval stage of about $50 \mathrm{~d}$, must resettle back on to mud to continue into the juvenile phase.

Fig. 5b illustrates a case in which larvae are released from a coastal spawning ground and are recruited to the juvenile stage at a distant 'downstream' coastal nursery area. This situation resembles the case of North Sea herring Clupea harengus. Herring larvae are released from spawning areas along the north-east coast of Britain and are carried by the general anti- 

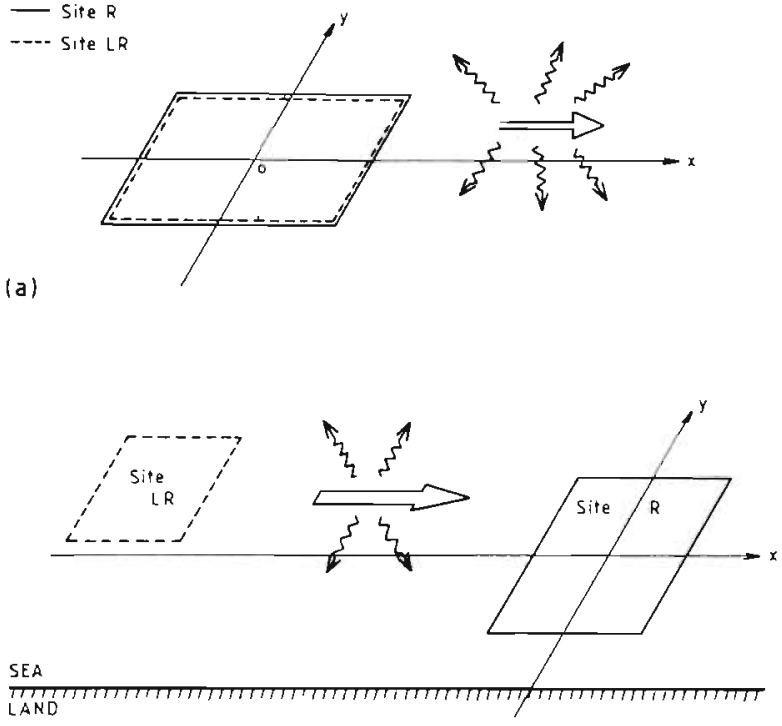

(b)

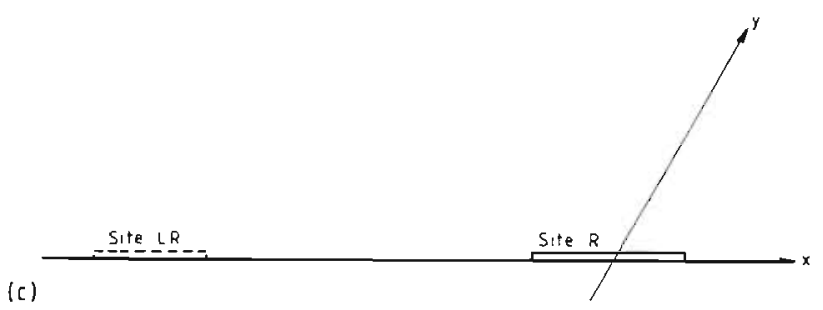

Fig. 5. A selection of idealised release and recruitment site configurations. (a) Release and recruitment site coincide. This configuration can be used to model larval release by an opensea, substrate-specific species, for example. (b) Release site is located upstream of a recruitment site close to a coast. (c) Release and recruitment sites are located along a segment of coastline. This may represent release and recruitment of estuarine species or of substrate-specific shore-line species

clockwise circulation of the North Sea to nursery areas along the coasts of Germany and Denmark. The dispersal of North Sea herring larvae by advection-diffusion processes was modelled in some detail by Bartsch et al. (1989) using a 3-dimensional hydrodynamic circulation model for the North Sea coupled to a random walk model for the diffusion process and a vertical migration model for larvae. Such numerical simulations are the best way to tackle the prediction of larval dispersal in particular instances (when resources are available) because they can account for realistic coastal geometry and bottom topography, as well as temporal and spatial variations in the advection and turbulence fields. The model described in this paper, by contrast, is intended for application to cases where such an approach may not be practicable. In such circumstances idealization of the real situation will be necessary for application of the solutions to obtain first order answers. Furthermore, by stripping the dispersal problem down to a bare minimum, the simple analytical solutions can be used to investigate the underlying principles of how dispersal influences population dynamics.

Fig. $5 c$ shows the case when the release site (LR) is stretched out into essentially a line segment along a portion of coastline. This could be used to represent release of larvae from the mouth of an estuary, say. It might also be applied to release of larvae from a segment of coastline such as a rocky shore. Similarly the recruitment sites ( $R$ ) may also be segments of shoreline again representing recruitment to estuaries or lengths of coastline with particular substrate properties.

The application of the solutions obtained above are somewhat restricted by the requirement that $u, v, K_{x}$, $K_{y}$ and $m$ be constant in order to obtain analytical results. The most serious implication of this is that (horizontal) spatial variabilty of the mean velocity $(u, v)$ and turbulence $\left(K_{x}, K_{y}\right)$ fields is not included. This limitation, therefore denies a detailed examination of larval concentration fields in response to particular spatially varying circulation structures such as closed gyres and eddies (e.g. Campana et al. 1989) or spatially varying mean flow fields (eg. Epifanio et al. 1989). As noted previously, these types of problems are best dealt with using numerical techniques which can incorporate spatial variability more readily.

\section{GENERAL RESULTS}

The results for spike releases and coastal releases are both special cases of releases from rectangular sites in an unbounded region. Results for unbounded sites (Eq. 15) are thus considered below. The cases when release site and recruitment site coincide and when release and recruitment sites are separated are both considered. For simplicity advection is assumed to take place in the $x$ direction only $(v=0)$ and the diffusion coefficient is set equal in all directions $\left(K_{x}=K_{y}=K\right)$. In the following cases release and recruitment sites both have dimensions $100 \mathrm{~km} \times 100 \mathrm{~km}$ and larval duration is taken as $\tau=50 \mathrm{~d}$.

\section{Relative contributions of advection and diffusion}

The fraction (as a percentage) of larvae that survive to the recruitment site, $\alpha=\left(N_{\tau} / N_{O}\right)$, with larval mortality, $m$, set to zero has been calculated from Eq. (15) for a range of values of advection and diffusion. Values of $\alpha=$ $\left(N_{\tau} / N_{0}\right)$ for a range of $u$ and $K$ are illustrated in Fig. 6a for the case where release and recruitment sites coincide. With increasing advection, $\alpha$ decreases sharply away 

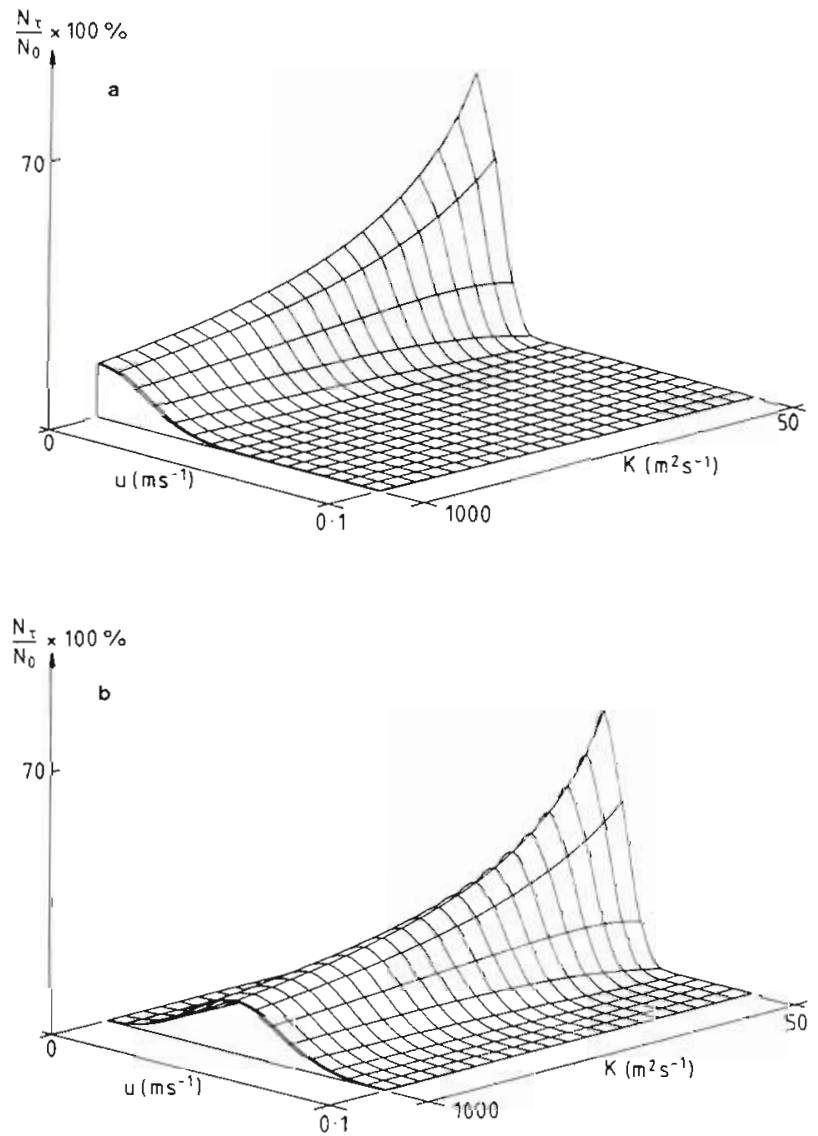

Fig. 6. Relative contributions of advection and diffusion to larval survival at a recruitment site. Horizontal axes show advection $u\left(\mathrm{~m} \mathrm{~s}^{-1}\right)$ and turbulent diffusion $\left(\mathrm{m}^{2} \mathrm{~s}^{-1}\right)$. The vertical axis shows percentage larval survival in the absence of mortality, $\alpha$. Recruitment and release sites both have dimensions $100 \mathrm{~km} \times 100 \mathrm{~km}$. Larval duration, $\tau$, is $50 \mathrm{~d}$. (a) Recruitment and release sites coincide. (b) Centre of recruitment site $300 \mathrm{~km}$ downstream from centre of release site

from its maximum at $u=0$. The decrease of $\alpha$ with increasing $K$ occurs much more slowly, however.

Fig. $6 \mathrm{~b}$ shows a case when the recruitment site is 300 $\mathrm{km}$ downstream of the release site. This figure again shows that percentage survival is highly sensitive to mean advection. For a given value of $u$, however, percentage survival changes rather slowly over the range of $K$. Peak survival in this example occurs for $u=$ $0.07 \mathrm{~m} \mathrm{~s}^{-1}$. For advection lower than this some larvae fail to reach the recruitment site and for higher advection larvae are carried beyond the recruitment site. The main point to note, however, is that significant changes in larval dispersal are brought about by changes in mean advection rather than by changes in turbulent diffusion. For this reason, in the following sections mean advection, $u$, is used as the primary measure of dispersal.

\section{Relative contributions of mortality and dispersal}

The percentage survival $\alpha=\left(N_{T} / N_{0}\right)$ for a range of values of dispersal (measured by $u$ ) and larval mortality, $m$, has been calculated using Eq. (15). Values of $\alpha$ for a range of $u$ and $m$ are shown in Fig. $7 \mathrm{a}$ for the case when release and recruitment sites coincide. Similarly Fig. $7 b$ shows the dependence of $\alpha$ upon $u$ and $m$ when
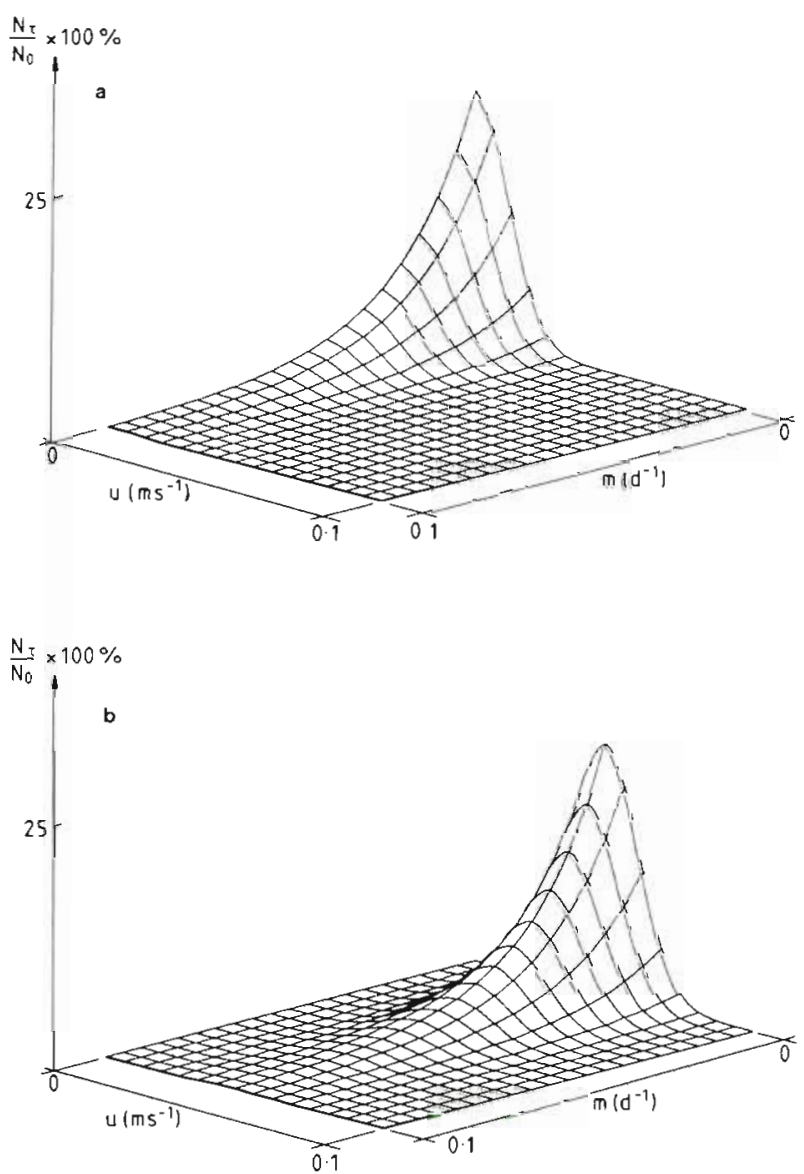

Fig. 7. Relative contributions of mortality and dispersal. Horizontal axes show advection $\left(u ; \mathrm{m} \mathrm{s}^{-1}\right.$ ) and larval mortality ( $m$; $\mathrm{d}^{-1}$ ). The vertical axis shows percentage larval survival $N_{r} / N_{0}$ Recruitment and release sites both have dimensions $100 \mathrm{~km} \times$ $100 \mathrm{~km}$. Larval duration, $\tau_{t}$ is $50 \mathrm{~d}$. (a) Recruitment and release sites coincide. (b) Centre of recruitment site $300 \mathrm{~km}$ downstream of centre of release site

the recruitment site is $300 \mathrm{~km}$ downstream of the release site. The point to note is that small changes in dispersal (u) have an effect upon survival equivalent to substantial changes in larval mortality. This result highlights the importance of dispersal processes, particularly advection, as a control upon the number of planktonic larvae surviving to the recruitment site. 


\section{Separation of release and recruitment sites}

Table 1 shows the percentage survival $\left(N_{\tau} / N_{0}\right)$ for a range of values of dispersal (measured by $u$ ) and a range of separation distances between release and recruitment sites. Larval mortality, $m$, is set to zero. Clearly, as separation of sites LR and R increases the optimal advection rate for ensuring maximum survival increases. When advection is too low, larvae fail to reach the recruitment site. If, on the other hand, advection is too large, larvae may be carried past the recruitment site.

\section{DISPERSAL AND FECUNDITY}

Long-term survival of a population depends upon sufficient recruitment of adults to replace losses due to mortality and vagrancy at all stages in the life-cycle. Suppose each year $N_{\mathrm{J}}$ organisms are recruited to the adult stock from juveniles. If $\tau_{\mathrm{A}}$ is the adult life-span and $m_{\mathrm{A}}$ is adult mortality then the total number of reproducing adults in the population is

$N_{\mathrm{a}}=N_{\mathrm{J}}\left(1-V_{\mathrm{A}}\right) \int_{0}^{\tau_{\mathrm{A}}} \exp \left(-m_{\mathrm{A}} t\right) \mathrm{d} t \approx \frac{\left(1-V_{\mathrm{A}}\right) N_{\mathrm{J}}}{m_{\mathrm{A}}}$

since $\exp \left(-m_{\mathrm{A}} \tau_{\mathrm{A}}\right) \approx 0$. In Eq. (25), $V_{\mathrm{A}}$ is adult vagrancy which accounts for all losses to the reproducing part of the adult stock other than by mortality. Vagrancy thus includes infertility, inability to find a mate and failure to return to the spawning site. If the reproducing part of the population is to be maintained in steady state, at a total of $N_{\mathrm{A}}$ adults, recruitment of juveniles at the level
$N_{j}$ must be maintained. If $N_{\tau}$ is the number of larvae that successfully reach the juvenile recruitment site, the number of juveniles produced from these larvae that survive to the end of the juvenile period (in order to be recruited to the adult stock is

$$
N_{\mathrm{J}}=N_{\tau}\left(1-V_{\mathrm{J}}\right) \exp \left(-m_{\mathrm{J}} \tau_{\mathrm{J}}\right)
$$

where $m_{J}=$ juvenile mortality; $\tau_{J}=$ duration of the juvenile phase; and $V_{\mathrm{J}}$ is the juvenile vagrancy which incorporates all juvenile losses other than mortality (such as wandering from the nursery area).

The $N_{\tau}$ larvae that survive to the juvenile recruitment site are related to the number of larvae hatched into the water column by

$$
N_{\mathrm{r}}=\left(1-V_{\mathrm{L}}\right) \exp (-m \tau) N_{0}
$$

where $N_{0}=$ number of larvae released from the spawning site and $V_{\mathrm{L}}=$ larval vagrancy [given by one of the results Eq. (10), Eq. (19) or Eq. (24)]. Finally $N_{0}$ is related to the number of reproductively active adults by

$$
N_{0}=\frac{\gamma N_{\mathrm{A}}}{2}
$$

where $\gamma=$ the effective female fecundity and an equal male-female sex ratio has been assumed. Substitution of Eqs. (26) to (28) into Eq. (25) gives the fecundity, $\gamma_{0}$, required to maintain the reproducing part of the adult population at a constant value $N_{\mathrm{A}}$

$$
\gamma_{0}=\frac{2 m_{\mathrm{A}} \exp \left(m_{\mathrm{J}} \tau_{\mathrm{J}}\right) \exp (m \tau)}{\left(1-V_{\mathrm{A}}\right)\left(1-V_{\mathrm{J}}\right)\left(1-\bar{V}_{\mathrm{L}}\right)}
$$

For $\gamma>\gamma_{0}$, population size will steadily increase until density-dependent control comes into operation. For

\begin{tabular}{|c|c|c|c|c|c|c|c|c|c|c|c|c|}
\hline \multirow{2}{*}{$D$} & \multicolumn{12}{|c|}{$u$} \\
\hline & 0 & 1 & 2 & 3 & 4 & 5 & 6 & 7 & 8 & 9 & 10 & 11 \\
\hline 0 & 26.2 & 22.5 & 14.3 & 6.6 & 2.2 & 0.5 & 0.1 & 0.0 & 0.0 & 0.0 & 0.0 & 0.0 \\
\hline 50 & 21.4 & 26.1 & 23.6 & 15.6 & 7.6 & 2.7 & 0.7 & 0.1 & 0.0 & 0.0 & 0.0 & 0.0 \\
\hline 100 & 11.6 & 20.2 & 25.8 & 24.4 & 17.0 & 8.7 & 3.2 & 0.8 & 0.2 & 0.0 & 0.0 & 0.0 \\
\hline 150 & 4.1 & 10.3 & 18.9 & 25.4 & 25.2 & 18.4 & 9.9 & 3.8 & 1.1 & 0.2 & 0.0 & 0.0 \\
\hline 200 & 0.9 & 3.4 & 9.1 & 17.5 & 24.7 & 25.7 & 19.7 & 11.1 & 4.5 & 1.3 & 0.3 & 0.0 \\
\hline 250 & 0.1 & 0.7 & 2.8 & 8.0 & 16.1 & 23.9 & 26.1 & 21.0 & 12.4 & 5.3 & 1.7 & 0.4 \\
\hline 300 & 0.0 & 0.1 & 0.6 & 2.3 & 6.9 & 14.7 & 22.9 & 26.2 & 22.2 & 13.8 & 6.2 & 2.0 \\
\hline 350 & 0.0 & 0.0 & 0.1 & 0.4 & 1.9 & 6.0 & 13.4 & 21.8 & 26.2 & 23.2 & 15.1 & 7.2 \\
\hline 400 & 0.0 & 0.0 & 0.0 & 0.1 & 0.3 & 1.6 & 5.1 & 12.0 & 20.6 & 26.0 & 24.1 & 16.5 \\
\hline 450 & 0.0 & 0.0 & 0.0 & 0.0 & 0.0 & 0.3 & 1.2 & 4.3 & 10.7 & 19.3 & 25.6 & 24.9 \\
\hline 500 & 0.0 & 0.0 & 0.0 & 0.0 & 0.0 & 0.0 & 0.2 & 1.0 & 3.6 & 9.5 & 18.0 & 25.0 \\
\hline 550 & 0.0 & 0.0 & 0.0 & 0.0 & 0.0 & 0.0 & 0.0 & 0.1 & 0.8 & 3.0 & 8.4 & 16.6 \\
\hline 600 & 0.0 & 0.0 & 0.0 & 0.0 & 0.0 & 0.0 & 0.0 & 0.0 & 0.1 & 0.6 & 2.5 & 7.3 \\
\hline 650 & 0.0 & 0.0 & 0.0 & 0.0 & 0.0 & 0.0 & 0.0 & 0.0 & 0.0 & 0.1 & 0.5 & 2.1 \\
\hline 700 & 0.0 & 0.0 & 0.0 & 0.0 & 0.0 & 0.0 & 0.0 & 0.0 & 0.0 & 0.0 & 0.1 & 0.4 \\
\hline 750 & 0.0 & 0.0 & 0.0 & 0.0 & 0.0 & 0.0 & 0.0 & 0.0 & 0.0 & 0.0 & 0.0 & 0.0 \\
\hline
\end{tabular}

Table 1. Percentage larval survival at the recruitment site for a range of mean advection $\left(u ; \mathrm{cm} \mathrm{s}^{-1}\right)$ and for a range of separation distances $\left(D_{i} \mathrm{~km}\right)$ between centres of release and recruitment sites. Larval mortality $m=0$. Release and recruitment sites both have dimensions $100 \mathrm{~km} \times 100 \mathrm{~km}$. Larval duration $\tau=50 \mathrm{~d}$ 
$\gamma<\gamma_{0}$, however, population size will steadily decline. Terms in the numerator of Eq. (29) are losses due to adult, juvenile and larval mortality respectively. Terms in the denominator incorporate all other forms of loss (vagrancy). Expressions for larval vagrancy, specifically, were derived in a previous section. Obviously Eq. (29) has the biologically important property that as mortality or vagrancy increases, the fecundity, $\gamma_{0}$, required to prevent population decline also increases. Note also that, when all forms of vagrancy are set to zero and when larval and juvenile mortality are also zero, $\gamma_{0}=2 \mathrm{~m}_{\mathrm{A}}$. In these circumstances the minimum fecundity is simply that required to replenish adult mortality losses (male plus female each with mortality $\left.m_{\mathrm{A}}\right)$.
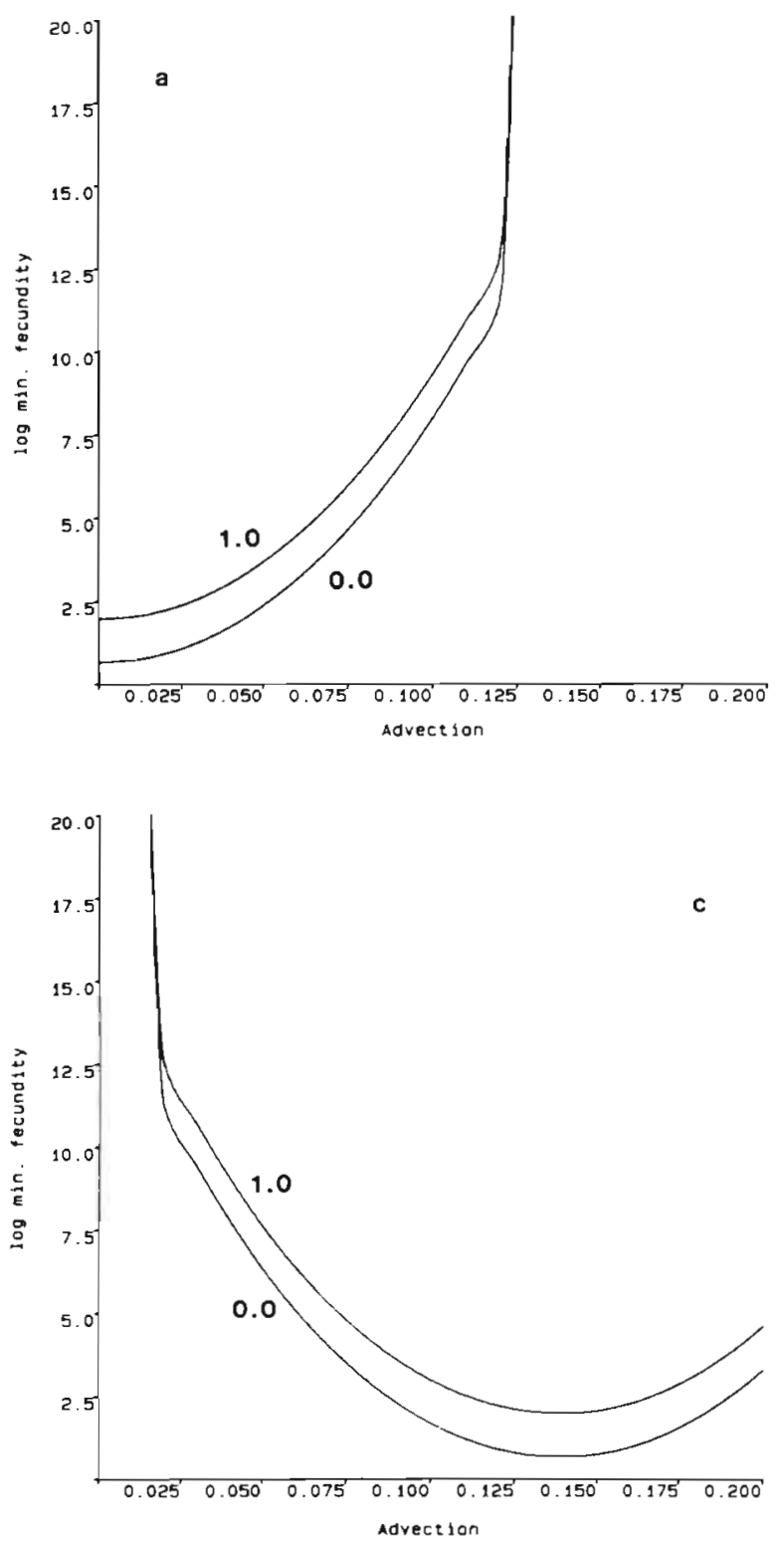

In the special case when all vagrancy is due to losses of larvae by advection-diffusion processes between release and recruitment sites (as is given by Eq. (19) say), the minimum fecundity, $\gamma_{0}$, below which the population cannot be sustained, is given by

$$
\gamma_{0}=\frac{2 D_{x} D_{y} m_{\mathrm{A}} \exp \left(m_{\mathrm{J}} \tau_{\mathrm{J}}\right) \exp (m \tau)}{\sqrt{K_{x} K_{y}} \tau F(\tau) G(\tau)}
$$

Fig. 8a shows $\gamma_{0}$ from Eq. (30) plotted against mean advection, $u$, for the case where release and recruitment sites coincide. Adult mortality is taken as $m_{\mathrm{A}}=0.6 \mathrm{yr}^{-1}$ and juvenile mortality, $m_{J}$, lies between 0 and $1.0 \mathrm{yr}^{-1}$. Horizontal diffusion is taken as $K_{x}=K_{y}=K=500 \mathrm{~m}^{2} \mathrm{~s}^{-1}$. Clearly as mean advection increases the fecundity required to ensure population survival increases also.

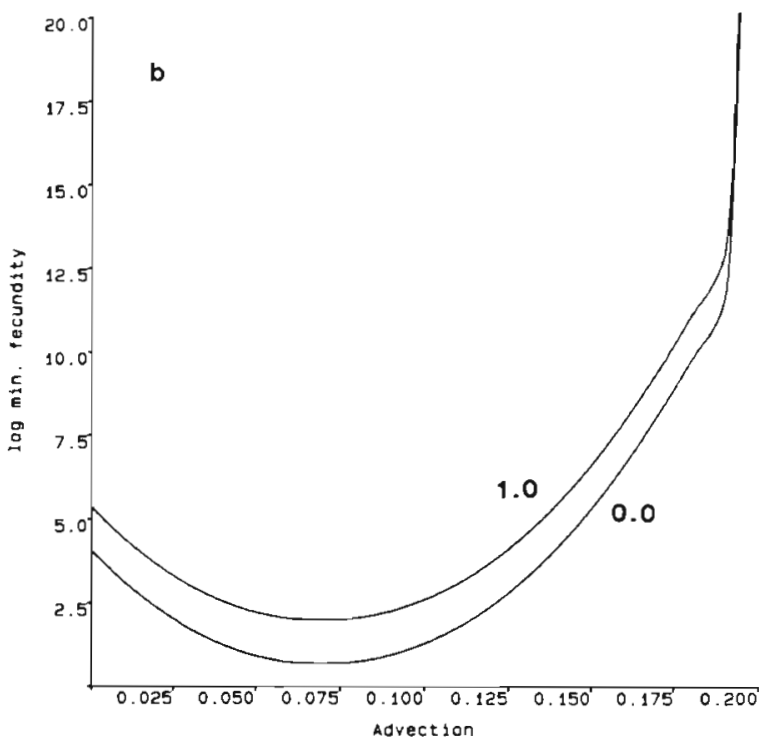

Fig. 8. Minimum fecundity, $\gamma_{0}$, required to maintain the reproducing part of the population at a constant level $N_{\mathrm{A}}$ for a range of values of advection $\left(u ; \mathrm{m} \mathrm{s}^{-1}\right)$ and juvenile mortality $\left(m_{\mathrm{Ji}}\right.$ $\mathrm{yr}^{-1}$ ) between 0 and $1.0 \mathrm{yr}^{-1}$. Recruitment and release sites both have dimensions $100 \mathrm{~km} \times 100 \mathrm{~km}$. Larval duration, $\tau_{\text {, }}$ is $50 \mathrm{~d}$ and juvenile duration $\tau_{\mathrm{J}}$ is $3 \mathrm{yr}$. (a) Recruitment site and release site coincide. (b) Recruitment site is $300 \mathrm{~km}$ downstream of the release site. (c) Recruitment site is $600 \mathrm{~km}$ downstream of the release site. Note in all cases the sharp cutoff for $\gamma_{0}$ which occurs when advection is either too high or too low for the population to be sustained against dispersive losses. Even before the point where the required fecundity becomes infinite, unrealistically high fecundities are predicted. In reality therefore the possible range of dispersion that a population can withstand is further restricted 
The minimum value of $\gamma_{0}$ for any $m_{j}$ occurs for zero advection. There is, however a critical value of advection, $\left(u=0.125 \mathrm{~m} \mathrm{~s}^{-1}\right)$, above which all larvae are swept away from the recruitment site and the required fecundity becomes infinite. Beyond this value of $u$, therefore, further increase in fecundity has no effect upon improving prospects for population survival.

Fig. $8 \mathrm{~b}$ shows the case when the centre of the recruitment site is $300 \mathrm{~km}$ downstream from the centre of the release site. Again, horizontal diffusion $K=500 \mathrm{~m}^{2} \mathrm{~s}^{-1}$. For any given $m_{\mathrm{J}}$ the lowest possible value of $\gamma_{0}$ occurs when $u$ maximizes the number of survivors at the recruitment site ( $u=0.07 \mathrm{~m} \mathrm{~s}^{-1}$ in this case). For $u>0.19 \mathrm{~m} \mathrm{~s}^{-1}$, however, further increase in fecundity cannot improve population survival as all larvae are advected past the recruitment site.

Fig. $8 \mathrm{c}$ shows the case when the recruitment site is $600 \mathrm{~km}$ downstream of the release site centre. For $u<0.02 \mathrm{~m} \mathrm{~s}^{-1}$, no larvae survive at the recruitment site irrespective of fecundity. Optimal fecundity occurs for $u=0.14 \mathrm{~m} \mathrm{~s}^{-1}$.

\section{DISCUSSION}

The advection-diffusion-mortality model described above is intended as a step towards quantifying the likely impact of pelagic larval losses upon recruitment. The basic components of the model are a spatially defined site from which larvae are released and a second well-defined site at which larvae must be located at the end of a planktonic period of a given duration. Between these sites advection-diffusion processes have been parameterised in the simplest possible form [constant advection $(u, v)$ and eddy diffusivity $\left.\left(K_{x}, K_{y}\right)\right]$ thus permitting relatively simple and general analytical solutions of the governing dispersal equation (Eq. 1).

The formulation of the model places emphasis upon the existence of 2 key sites which place spatial constraints upon life-cycle closure. The importance of such constraints upon life-cycle continuity is also central to the member/vagrant hypothesis of Sinclair (1988) and Sinclair \& Iles (1989). The present study thus expresses in mathematical terms parts of the member/vagrant hypothesis.

The first site of importance is the recruitment site. The transition from a planktonic larval phase to a juvenile stage is generally accompanied by changes in feeding and habitat requirements as well as upon the pattern of predation for the organism. Commonly these changes are reflected by a need to be located in a more restricted region of space (which has been termed the recruitment site) where the new set of requirements can be satisfied at the time of transition. The first major challenge in life-cycle closure, therefore, is to reach the recruitment site. Although an organism may be perfectly successful during its planktonic larval stage (by avoiding predation and being located in a region rich in food supply), this success will be of no consequence if the organism fails to reach a suitable recruitment site where the different requirements of the next stage of its life-cycle can be satisfied.

The second major challenge in life-cycle closure is for adults to return to a breeding site at a suitable time. Organisms which fail to do this (or are ineffective breeders when they get there) are termed adult vagrants and do not pass genetic material into the next generation. In evolutionary terms, genes which promote a systematic tendency towards vagrancy in a population will be selected against. Usually the adult breeding site is equivalent to the site for larval release. Thus the recruitment and release sites which form the basis of the model are both critical life-cycle closure sites. The physical processes of advection-diffusion play an important role in determining which organisms reach the recruitment site. In contrast, return of adults to the release site is probably influenced to a greater degree by biological processes

The model presented here incorporates a basic strategy for dealing with the problem of quantifying larval vagrancy as part of wider recruitment and population regulation problems. Because of its simplicity, however, the model lacks many of the refinements needed for application in specific cases. An obvious next stage in model improvement is to incorporate realistic spatial and temporal variability of advectiondiffusion processes. This will, however, necessarily involve the adoption of a numerical modelling approach to permit a move towards results of more sitespecific interest.

A general difficulty associated with the approach adopted in the present model concerns the way in which the location of the recruitment site is specified. In the present treatment the recruitment site is rigidly defined as a rectangular region and only larvae that are dispersed into that rectangle are recruited. In reality, of course, many organisms, particularly in their later stages of larval development, have a limited capacity for independent horizontal motion or alternatively may use systematic vertical migration in relation to current shear to control horizontal movement. In these circumstances the boundaries of the recruitment site should probably be viewed as being rather 'fuzzy' reflecting the operation of active as well as passive transport processes in the vicinity of the recruitment site. This point raises a general question about the way in which pelagic larvae use their abilities to actively control position. Are such mechanisms used extensively throughout the pelagic phase (at long range from a 
recruitment site) or are they essentially used for 'fine tuning' the final approach in relatively close proximity to the recruitment site? There is evidence from a number of species of active vertical migration and siteselection behaviour near recruitment sites (e.g. Butman 1987. Christy 1989). If such activity is indeed largely confined to recruitment site approaches, then advection-diffusion models may provide a good description. of larval distributions over much of the pelagic phase.

\section{LITERATURE CITED}

Bartsch, J., Brander, K., Heath, M., Munk, P., Richardson, K., Svendsen, E. (1989). Modelling advection of herring larvae in the North Sea. Nature, Lond. 340: 632-636

Beverton, R. J. H., Holt, S. J. (1957). On the dynamics of exploited fish populations. Fishery Investigations. Series 2 , Vol. 19. Ministry of Agriculture Fisheries and Food. Her Majesty's Stationery Office, London

Blaxter, J. H. S., Gamble, J. C., Westernhagen, H. von (eds.) (1989). The early life history of fish. Rapp. P-v. Réun. Cons. int Explor. Mer. 191

Butman, C. A. (1987). Larval settlement of soft sediment invertebrates: the spatial scales of pattern explained by active habitat selection and the emerging role of hydrodynamical processes. Oceanogr. mar. Biol. A. Rev. 25: 113-165

Campana, S. E., Smith, S. J., Hurley, P. C. F. (1989). An agestructured index of cod larval drift and retention in the

This article was presented by E. Naylor, Menai Bridge, United Kingdom waters off southwest Nova Scotia. Rapp P.-v. Réun. Cons. int. Explor. Mer 191: 50-62

Christy, J. H. (1989). Rapid development of megalopae of fiddler crab Uca pugilator reared over sediment: implications for models of larval recruitment. Mar. Ecol. Prog. Ser 57: 259-265

Epifanio, C. E., Masse, A. K., Garvine, R. W. (1989). Transport of blue crab larvae by surface currents off Delaware Bay, USA. Mar. Ecol. Prog. Ser. 54: 35-41

Fischer, H. B., List, E. J., Koh, R. C. Y., Imberger, J., Brooks, N H. (1979). Mixing in coastal and inland waters. Academic Press, London

Goodrich, D. M., van Montfrans, J., Orth, R. J. (1989). Blue crab megalopal influx to Chesapeake Bay: evidence for a winddriven mechanism. Estuar. coast. Shelf Sci. 29: 247-260

Hill, A. E. (1990). Pelagic dispersal of Norway lobster Nephrops norvegicus larvae examined using an advection-diffusion-mortality model. Mar. Ecol. Prog. Ser. 64: 217-226

Koutsikopoulos, C., Desaunay, Y., Dorel, D., Marchand, J. (1989). The role of coastal areas in the life-history of Sole (Solea solea L.) in the Bay of Biscay. In: Ros, J. D. (ed.) Topics in marine biology. Scientia mar. (spec. issue) 53 : $567-575$

Sinclair, M. (1988). Marine populations: an essay on population regulation and speciation. Washington Sea Grant/ University of Washington Press, Seattle

Sinclair, M., Iles, T. D. (1989). Population regulation and speciation in the oceans. J. Cons. int. Explor. Mer 45: 165-175

Veer, H. W van der, Pihl, L., Bergman, M. J. N. (1990). Recruitment mechanisms in North Sea plaice Pleuronectes platessa. Mar. Ecol. Prog. Ser. 64: 1-12

Manuscript first received: September 6, 1990

Revised version accepted: December 5, 1990 